Article

\title{
On a Dual Pair of Multiobjective Interval-Valued Variational Control Problems
}

\author{
Savin Treanţă (D)
}

check for

updates

Citation: Treanţă, S. On a Dual Pair of Multiobjective Interval-Valued Variational Control Problems. Mathematics 2021, 9, 893. https:// doi.org/10.3390/math9080893

Academic Editor: Aleksandr Rakhmangulov

Received: 7 April 2021

Accepted: 15 April 2021

Published: 17 April 2021

Publisher's Note: MDPI stays neutral with regard to jurisdictional claims in published maps and institutional affiliations.

Copyright: (C) 2021 by the author. Licensee MDPI, Basel, Switzerland. This article is an open access article distributed under the terms and conditions of the Creative Commons Attribution (CC BY) license (https:// creativecommons.org/licenses/by/ $4.0 /)$.
Department of Applied Mathematics, University Politehnica of Bucharest, 060042 Bucharest, Romania; savin.treanta@upb.ro

\begin{abstract}
In this paper, by using the new concept of $(\varrho, \psi, \omega)$-quasiinvexity associated with intervalvalued path-independent curvilinear integral functionals, we establish some duality results for a new class of multiobjective variational control problems with interval-valued components. More concretely, we formulate and prove weak, strong, and converse duality theorems under $(\varrho, \psi, \omega)$ quasiinvexity hypotheses for the considered class of optimization problems.
\end{abstract}

Keywords: multiobjective optimization problem; LU-efficiency conditions; $(\varrho, \psi, \omega)$-quasiinvexity; interval-valued path-independent curvilinear integral functional; duality; control

\section{Introduction}

Duality theory represents an important part in the study of mathematical programming problems. Due to its effectiveness, it has been extended and generalized to new classes of optimization problems. Here, we mention the classical research papers of Hanson [1], Mond and Hanson [2], Mond and Smart [3], Aggarwal et al. [4]. Further, the multiobjective optimization problems with mixed constraints have been studied by many researchers, with remarkable results. In this regard, Mishra and Mukherjee [5] considered a multiobjective control problem and established Mond-Weir duality results under V-invexity assumptions and their generalizations. Ahmad and Sharma [6] obtained sufficient conditions of optimality and formulated Wolfe and Mond-Weir duals for a class of multiobjective variational control problems. Further, Antczak [7] established Mond-Weir and Wolfe type duals for multiobjective variational control problems under $(\Phi, \rho)$-invexity. Recently, Mititelu and Treanţă [8] formulated and proved efficiency conditions in vector control problems governed by multiple integrals. Following this work, Treanţă [9] investigated the necessary and sufficient efficiency conditions in uncertain variational control problems. For more and various contributions and approaches to multiobjective variational control problems, the reader is directed to Zhian and Qingkai [10], Mititelu [11], Treanţă and Udrişte [12], Zalmai [13], Hachimi and Aghezzaf [14], Treanţă [15,16], Treanţă and Mititelu [17], Chen [18], Kim and Kim [19], Gulati et al. [20], Nahak and Nanda [21], Arana-Jiménez et al. [22], Khazafi et al. [23], Zhang et al. [24], Treanţă and Arana-Jiménez [25].

The present paper, motivated by the aforementioned research works and practical reasons, establishes weak, strong, and converse Mond-Weir duality results for a new class of multiobjective optimization problems with interval-valued components governed by path-independent curvilinear integral functionals. The main novelty elements of this work are represented by the necessary LU-efficiency conditions derived using some recent research papers of the author; the notion of $(\varrho, \psi, \omega)$-quasiinvexity associated with intervalvalued path-independent curvilinear integral functionals; and the presence of a partition associated with a set of indices used for the inequality-type constraints.

In the following, we organize the paper as follows: in Section 2, we present notations, preliminary mathematical tools, and the problem formulation we are going to study; in Section 3, we establish the main results of this paper-namely, weak, strong, and con- 
verse Mond-Weir dualities are formulated and proved for the new class of multiobjective optimization problems; finally, in Section 4, we conclude the paper.

\section{Preliminaries}

Throughout this paper, we consider $\Omega$ as a compact domain in the Euclidean real space $\mathbb{R}^{m}$ and denote by $t=\left(t^{\alpha}\right), \alpha=\overline{1, m}, u=\left(u^{j}\right), j \in \overline{1, k}$, and $a=\left(a^{i}\right), i=\overline{1, n}$, the points in $\Omega, \mathbb{R}^{k}$, and $\mathbb{R}^{n}$, respectively. Further, consider that $\Omega \supset \gamma$ is a piecewise smooth curve joining the different points $t_{1}=\left(t_{1}^{1}, \ldots, t_{1}^{m}\right), t_{2}=\left(t_{2}^{1}, \ldots, t_{2}^{m}\right)$ in $\Omega$. Now, we define the following continuously differentiable functions

$$
\begin{gathered}
\mathcal{X}=\left(\mathcal{X}_{\alpha}^{i}\right): \Omega \times \mathbb{R}^{n} \times \mathbb{R}^{k} \rightarrow \mathbb{R}^{n m}, \quad i=\overline{1, n}, \alpha=\overline{1, m}, \\
\mathcal{Y}=\left(\mathcal{Y}_{1}, \ldots, \mathcal{Y}_{q}\right)=\left(\mathcal{Y}_{\beta}\right): \Omega \times \mathbb{R}^{n} \times \mathbb{R}^{k} \rightarrow \mathbb{R}^{q}, \quad \beta=\overline{1, q},
\end{gathered}
$$

and we accept that the following Lagrange densities

$$
\mathcal{X}_{\alpha}=\left(\mathcal{X}_{\alpha}^{i}\right): \mathcal{P} \rightarrow \mathbb{R}^{n}, \quad i=\overline{1, n}, \alpha=\overline{1, m},
$$

satisfy the closeness conditions (complete integrability conditions)

$$
D_{\eta} \mathcal{X}_{\alpha}^{i}=D_{\alpha} \mathcal{X}_{\eta,}^{i} \quad \alpha, \eta=\overline{1, m}, \alpha \neq \eta, i=\overline{1, n},
$$

where $D_{\eta}$ is the total derivative operator. Denote by $\mathcal{A}$ the space of all piecewise smooth state functions $a: \Omega \rightarrow \mathbb{R}^{n}$, and by $\mathcal{U}$ the space of all piecewise continuous control functions $u: \Omega \rightarrow \mathbb{R}^{k}$, endowed with the induced norm. Additionally, in this paper, for any two $p$-tuples $l=\left(l_{1}, \ldots, l_{p}\right), c=\left(c_{1}, \ldots, c_{p}\right)$ in $\mathbb{R}^{p}$, we use the following partial ordering

$$
\begin{gathered}
l=c \Leftrightarrow l_{r}=c_{r}, \quad l \leq c \Leftrightarrow l_{r} \leq c_{r}, \\
l<c \Leftrightarrow l_{r}<c_{r}, \quad l \preceq c \Leftrightarrow l \leq c, l \neq c, \quad r=\overline{1, p} .
\end{gathered}
$$

Let $K$ be the set of all closed and bounded real intervals. Denote by $I=\left[i^{L},{ }_{i}{ }^{U}\right]$ a closed and bounded real interval, where $i^{L}$ and $i^{U}$ indicate the lower and upper bounds of $I$, respectively. Throughout this paper, the interval operations are performed as follows:

(1) $I=J \Longrightarrow i^{L}=j^{L}$ and $i^{U}=j^{U}$;

(2) if $i^{L}=i^{U}=i$ then $I=[i, i]=i$;

(3) $I+J=\left[i^{L}+j^{L}, i^{U}+j^{U}\right]$;

(4) $-I=-\left[i^{L}, i^{U}\right]=\left[-i^{U},-i^{L}\right]$;

(5) $I-J=\left[i^{L}-j^{U}, i^{U}-j^{L}\right]$;

(6) $k+I=\left[k+i^{L}, k+i^{U}\right], k \in \mathbb{R}$;

(7) $k A=\left[k a^{L}, k a^{U}\right], k \in \mathbb{R}, k \geq 0$;

$\left(7^{\prime}\right) k A=\left[k a^{U}, k a^{L}\right], k \in \mathbb{R}, k<0$;

(8) $I / J=\left[i^{L} / j^{L}, i^{U} / j^{U}\right], j^{L}, J^{U}>0$.

Definition 1 (Treanţă [9]). Let $I, J \in K$. We write $I \leq J$ if and only if $i^{L} \leq j^{L}$ and $i^{U} \leq j^{U}$. Further, we write $I<J$ if and only if $i^{L}<j^{L}$ and $i^{U}<j^{U}$.

Definition 2 (Treanţă [9]). A function $f: \Omega \times \mathbb{R}^{n} \times \mathbb{R}^{k} \rightarrow K$, defined by

$$
f(t, a(t), u(t))=\left[f^{L}(t, a(t), u(t)), f^{U}(t, a(t), u(t))\right], \quad t \in \Omega,
$$

where $f^{L}(t, a(t), u(t))$ and $f^{U}(t, a(t), u(t))$ are real-valued functions and satisfy the condition $f^{L}(t, a(t), u(t)) \leq f^{U}(t, a(t), u(t)) ; t \in \Omega$ is said to be an interval-valued function. 
For $\alpha=\overline{1, m}$, we consider the following vector continuously differentiable functions with interval-valued components (closed 1-forms)

$$
\begin{aligned}
& f_{\alpha}=\left(f_{\alpha}^{1}, \ldots, f_{\alpha}^{p}\right)=\left(f_{\alpha}^{r}\right): \Omega \times \mathbb{R}^{n} \times \mathbb{R}^{k} \rightarrow K^{p}, \quad r=\overline{1, p} \\
& g_{\alpha}=\left(g_{\alpha}^{1}, \ldots, g_{\alpha}^{p}\right)=\left(g_{\alpha}^{r}\right): \Omega \times \mathbb{R}^{n} \times \mathbb{R}^{k} \rightarrow K^{p}, \quad r=\overline{1, p},
\end{aligned}
$$

which, for $r=\overline{1, p}$, generate the interval-valued path-independent curvilinear integral functionals (see Einstein summation):

$$
\begin{gathered}
F^{r}, G^{r}: \mathcal{A} \times \mathcal{U} \rightarrow K, \\
F^{r}(a, u)=\int_{\gamma} f_{\alpha}^{r}(t, a(t), u(t)) d t^{\alpha}=\left[\int_{\gamma} f_{\alpha}^{r, L}(t, a(t), u(t)) d t^{\alpha}, \int_{\gamma} f_{\alpha}^{r, U}(t, a(t), u(t)) d t^{\alpha}\right] \\
G^{r}(a, u)=\int_{\gamma} g_{\alpha}^{r}(t, a(t), u(t)) d t^{\alpha}=\left[\int_{\gamma} g_{\alpha}^{r, L}(t, a(t), u(t)) d t^{\alpha}, \int_{\gamma} g_{\alpha}^{r, U}(t, a(t), u(t)) d t^{\alpha}\right] .
\end{gathered}
$$

Further, in accordance with Treanţă and Mititelu [17,26], following Treanţă [9], in order to formulate and prove the main results included in this paper, we introduce the concept of $(\varrho, \psi, \omega)$-quasiinvexity associated with an interval-valued path-independent curvilinear integral functional. For $\alpha=\overline{1, m}$, we consider an interval-valued continuously differentiable function:

$$
\begin{gathered}
h_{\alpha}: \Omega \times \mathbb{R}^{n} \times \mathbb{R}^{n m} \times \mathbb{R}^{k} \rightarrow K, \\
h_{\alpha}=h_{\alpha}\left(t, a(t), a_{\zeta}(t), u(t)\right)=\left[h_{\alpha}^{L}\left(t, a(t), a_{\zeta}(t), u(t)\right), h_{\alpha}^{U}\left(t, a(t), a_{\zeta}(t), u(t)\right)\right],
\end{gathered}
$$

where $a_{\zeta}(t):=\frac{\partial a}{\partial t}(t)$ and for $a \in \mathcal{A}$ and $u \in \mathcal{U}$, we introduce the following interval-valued path-independent curvilinear integral functional:

$$
\begin{aligned}
& H: \mathcal{A} \times \mathcal{U} \rightarrow K, \quad H(a, u)=\int_{\gamma} h_{\alpha}\left(t, a(t), a_{\zeta}(t), u(t)\right) d t^{\alpha} \\
= & {\left[\int_{\gamma} h_{\alpha}^{L}\left(t, a(t), a_{\zeta}(t), u(t)\right) d t^{\alpha}, \int_{\gamma} h_{\alpha}^{U}\left(t, a(t), a_{\zeta}(t), u(t)\right) d t^{\alpha}\right] . }
\end{aligned}
$$

Furthermore, let $\varrho$ be a real number, $\psi: \mathcal{A} \times \mathcal{U} \times \mathcal{A} \times \mathcal{U} \rightarrow[0, \infty)$ be a positive functional, and $\omega\left((a, u),\left(a^{0}, u^{0}\right)\right)$ be a real-valued function on $(\mathcal{A} \times \mathcal{U})^{2}$.

\section{Definition 3.}

(i) If there exist

$$
v=v\left(t, a(t), u(t), a^{0}(t), u^{0}(t)\right)=\left(v_{i}\left(t, a(t), u(t), a^{0}(t), u^{0}(t)\right)\right), \quad i=1, \ldots, n,
$$

of $C^{1}$-class with $v\left(t, a^{0}(t), u^{0}(t), a^{0}(t), u^{0}(t)\right)=0, \forall t \in \Omega, v\left(t_{1}\right)=v\left(t_{2}\right)=0$, and

$$
\begin{gathered}
\tau: \Omega \times \mathbb{R}^{n} \times \mathbb{R}^{k} \times \mathbb{R}^{n} \times \mathbb{R}^{k} \rightarrow \mathbb{R}^{k}, \\
\tau=\tau\left(t, a(t), u(t), a^{0}(t), u^{0}(t)\right)=\left(\tau_{j}\left(t, a(t), u(t), a^{0}(t), u^{0}(t)\right)\right), \quad j=1, \ldots, k,
\end{gathered}
$$

of $C^{0}$-class with $\tau\left(t, a^{0}(t), u^{0}(t), a^{0}(t), u^{0}(t)\right)=0, \forall t \in \Omega, \tau\left(t_{1}\right)=\tau\left(t_{2}\right)=0$, such that for every $(a, u) \in \mathcal{A} \times \mathcal{U}$,

$$
\begin{gathered}
H(a, u) \leq H\left(a^{0}, u^{0}\right) \\
\Rightarrow \psi\left(a, u, a^{0}, u^{0}\right) \int_{\gamma}\left[\left(h_{\alpha}^{L}\right)_{a}\left(t, a^{0}(t), a_{\zeta}^{0}(t), u^{0}(t)\right),\left(h_{\alpha}^{U}\right)_{a}\left(t, a^{0}(t), a_{\zeta}^{0}(t), u^{0}(t)\right)\right] v d t^{\alpha}
\end{gathered}
$$




$$
\begin{gathered}
+\psi\left(a, u, a^{0}, u^{0}\right) \int_{\gamma}\left[\left(h_{\alpha}^{L}\right)_{a_{\zeta}}\left(t, a^{0}(t), a_{\zeta}^{0}(t), u^{0}(t)\right),\left(h_{\alpha}^{U}\right)_{a_{\zeta}}\left(t, a^{0}(t), a_{\zeta}^{0}(t), u^{0}(t)\right)\right] D_{\zeta} v d t^{\alpha} \\
+\psi\left(a, u, a^{0}, u^{0}\right) \int_{\gamma}\left[\left(h_{\alpha}^{L}\right)_{u}\left(t, a^{0}(t), a_{\zeta}^{0}(t), u^{0}(t)\right),\left(h_{\alpha}^{U}\right)_{u}\left(t, a^{0}(t), a_{\zeta}^{0}(t), u^{0}(t)\right)\right] \tau d t^{\alpha} \\
+\varrho \psi\left(a, u, a^{0}, u^{0}\right) \omega^{2}\left((a, u),\left(a^{0}, u^{0}\right)\right) \leq[0,0],
\end{gathered}
$$

or, equivalently,

$$
\begin{gathered}
\psi\left(a, u, a^{0}, u^{0}\right) \int_{\gamma}\left[\left(h_{\alpha}^{L}\right)_{a}\left(t, a^{0}(t), a_{\zeta}^{0}(t), u^{0}(t)\right),\left(h_{\alpha}^{U}\right)_{a}\left(t, a^{0}(t), a_{\zeta}^{0}(t), u^{0}(t)\right)\right] v d t^{\alpha} \\
+\psi\left(a, u, a^{0}, u^{0}\right) \int_{\gamma}\left[\left(h_{\alpha}^{L}\right)_{a_{\zeta}}\left(t, a^{0}(t), a_{\zeta}^{0}(t), u^{0}(t)\right),\left(h_{\alpha}^{U}\right)_{a_{\zeta}}\left(t, a^{0}(t), a_{\zeta}^{0}(t), u^{0}(t)\right)\right] D_{\zeta} v d t^{\alpha} \\
+\psi\left(a, u, a^{0}, u^{0}\right) \int_{\gamma}\left[\left(h_{\alpha}^{L}\right)_{u}\left(t, a^{0}(t), a_{\zeta}^{0}(t), u^{0}(t)\right),\left(h_{\alpha}^{U}\right)_{u}\left(t, a^{0}(t), a_{\zeta}^{0}(t), u^{0}(t)\right)\right] \tau d t^{\alpha} \\
+\varrho \psi\left(a, u, a^{0}, u^{0}\right) \omega^{2}\left((a, u),\left(a^{0}, u^{0}\right)\right)>[0,0] \Rightarrow H(a, u)>H\left(a^{0}, u^{0}\right),
\end{gathered}
$$

then, $H$ is said to be $(\varrho, \psi, \omega)$-quasiinvex at $\left(a^{0}, u^{0}\right) \in \mathcal{A} \times \mathcal{U}$ with respect to $v$ and $\tau$.

(ii) If there exist

$$
v: \Omega \times \mathbb{R}^{n} \times \mathbb{R}^{k} \times \mathbb{R}^{n} \times \mathbb{R}^{k} \rightarrow \mathbb{R}^{n},
$$

$$
v=v\left(t, a(t), u(t), a^{0}(t), u^{0}(t)\right)=\left(v_{i}\left(t, a(t), u(t), a^{0}(t), u^{0}(t)\right)\right), \quad i=1, \ldots, n,
$$

of $C^{1}$-class with $v\left(t, a^{0}(t), u^{0}(t), a^{0}(t), u^{0}(t)\right)=0, \forall t \in \Omega, v\left(t_{1}\right)=v\left(t_{2}\right)=0$, and

$$
\begin{gathered}
\tau: \Omega \times \mathbb{R}^{n} \times \mathbb{R}^{k} \times \mathbb{R}^{n} \times \mathbb{R}^{k} \rightarrow \mathbb{R}^{k}, \\
\tau=\tau\left(t, a(t), u(t), a^{0}(t), u^{0}(t)\right)=\left(\tau_{j}\left(t, a(t), u(t), a^{0}(t), u^{0}(t)\right)\right), \quad j=1, \ldots, k,
\end{gathered}
$$

of $C^{0}$-class with $\tau\left(t, a^{0}(t), u^{0}(t), a^{0}(t), u^{0}(t)\right)=0, \forall t \in \Omega, \tau\left(t_{1}\right)=\tau\left(t_{2}\right)=0$, such that for every $(a, u) \neq\left(a^{0}, u^{0}\right) \in \mathcal{A} \times \mathcal{U}$,

$$
\begin{gathered}
H(a, u) \leq H\left(a^{0}, u^{0}\right) \\
\Rightarrow \psi\left(a, u, a^{0}, u^{0}\right) \int_{\gamma}\left[\left(h_{\alpha}^{L}\right)_{a}\left(t, a^{0}(t), a_{\zeta}^{0}(t), u^{0}(t)\right),\left(h_{\alpha}^{U}\right)_{a}\left(t, a^{0}(t), a_{\zeta}^{0}(t), u^{0}(t)\right)\right] v d t^{\alpha} \\
+\psi\left(a, u, a^{0}, u^{0}\right) \int_{\gamma}\left[\left(h_{\alpha}^{L}\right)_{a_{\zeta}}\left(t, a^{0}(t), a_{\zeta}^{0}(t), u^{0}(t)\right),\left(h_{\alpha}^{U}\right)_{a_{\zeta}}\left(t, a^{0}(t), a_{\zeta}^{0}(t), u^{0}(t)\right)\right] D_{\zeta} v d t^{\alpha} \\
+\psi\left(a, u, a^{0}, u^{0}\right) \int_{\gamma}\left[\left(h_{\alpha}^{L}\right)_{u}\left(t, a^{0}(t), a_{\zeta}^{0}(t), u^{0}(t)\right),\left(h_{\alpha}^{U}\right)_{u}\left(t, a^{0}(t), a_{\zeta}^{0}(t), u^{0}(t)\right)\right] \tau d t^{\alpha} \\
+\varrho \psi\left(a, u, a^{0}, u^{0}\right) \omega^{2}\left((a, u),\left(a^{0}, u^{0}\right)\right)<[0,0],
\end{gathered}
$$

or, equivalently,

$$
\begin{gathered}
\psi\left(a, u, a^{0}, u^{0}\right) \int_{\gamma}\left[\left(h_{\alpha}^{L}\right)_{a}\left(t, a^{0}(t), a_{\zeta}^{0}(t), u^{0}(t)\right),\left(h_{\alpha}^{U}\right)_{a}\left(t, a^{0}(t), a_{\zeta}^{0}(t), u^{0}(t)\right)\right] v d t^{\alpha} \\
+\psi\left(a, u, a^{0}, u^{0}\right) \int_{\gamma}\left[\left(h_{\alpha}^{L}\right)_{a_{\zeta}}\left(t, a^{0}(t), a_{\zeta}^{0}(t), u^{0}(t)\right),\left(h_{\alpha}^{U}\right)_{a_{\zeta}}\left(t, a^{0}(t), a_{\zeta}^{0}(t), u^{0}(t)\right)\right] D_{\zeta} v d t^{\alpha} \\
+\psi\left(a, u, a^{0}, u^{0}\right) \int_{\gamma}\left[\left(h_{\alpha}^{L}\right)_{u}\left(t, a^{0}(t), a_{\zeta}^{0}(t), u^{0}(t)\right),\left(h_{\alpha}^{U}\right)_{u}\left(t, a^{0}(t), a_{\zeta}^{0}(t), u^{0}(t)\right)\right] \tau d t^{\alpha} \\
+\varrho \psi\left(a, u, a^{0}, u^{0}\right) \omega^{2}\left((a, u),\left(a^{0}, u^{0}\right)\right) \geq[0,0] \Rightarrow H(a, u)>H\left(a^{0}, u^{0}\right),
\end{gathered}
$$


then, $H$ is said to be strictly $(\varrho, \psi, \omega)$-quasiinvex at $\left(a^{0}, u^{0}\right) \in \mathcal{A} \times \mathcal{U}$ with respect to $v$ and $\tau$.

Next, for $\alpha=\overline{1, m}$, we consider the vector continuously differentiable function with interval-valued components

$$
\begin{gathered}
h_{\alpha}: \Omega \times \mathbb{R}^{n} \times \mathbb{R}^{n m} \times \mathbb{R}^{k} \rightarrow K^{p}, \\
h_{\alpha}=h_{\alpha}\left(t, a(t), a_{\zeta}(t), u(t)\right)=\left(h_{\alpha}^{r}\left(t, a(t), a_{\zeta}(t), u(t)\right)\right) \\
=\left(\left[h_{\alpha}^{1, L}\left(t, a(t), a_{\zeta}(t), u(t)\right), h_{\alpha}^{1, U}\left(t, a(t), a_{\zeta}(t), u(t)\right)\right],\right. \\
\left.\ldots,\left[h_{\alpha}^{p, L}\left(t, a(t), a_{\zeta}(t), u(t)\right), h_{\alpha}^{p, U}\left(t, a(t), a_{\zeta}(t), u(t)\right)\right]\right) .
\end{gathered}
$$

Definition 4. The vector path-independent curvilinear integral functional with interval-valued components

$$
\begin{gathered}
H: \mathcal{A} \times \mathcal{U} \rightarrow K^{p}, \quad H(a, u)=\int_{\gamma} h_{\alpha}\left(t, a(t), a_{\zeta}(t), u(t)\right) d t^{\alpha} \\
=\left(\left[\int_{\gamma} h_{\alpha}^{1, L}\left(t, a(t), a_{\zeta}(t), u(t)\right) d t^{\alpha}, \int_{\gamma} h_{\alpha}^{1, U}\left(t, a(t), a_{\zeta}(t), u(t)\right) d t^{\alpha}\right],\right. \\
\left.\cdots,\left[\int_{\gamma} h_{\alpha}^{p, L}\left(t, a(t), a_{\zeta}(t), u(t)\right) d t^{\alpha}, \int_{\gamma} h_{\alpha}^{p, U}\left(t, a(t), a_{\zeta}(t), u(t)\right) d t^{\alpha}\right]\right)
\end{gathered}
$$

is said to be $(\varrho, \psi, \omega)$-quasiinvex (strictly $(\varrho, \psi, \omega)$-quasiinvex) at $\left(a^{0}, u^{0}\right) \in \mathcal{A} \times \mathcal{U}$ with respect to $v$ and $\tau$, if each interval-valued component of the vector is $(\varrho, \psi, \omega)$-quasiinvex (strictly $(\varrho, \psi, \omega)$ quasiinvex) at $\left(a^{0}, u^{0}\right) \in \mathcal{A} \times \mathcal{U}$ with respect to $v$ and $\tau$.

Now, we are in a position to formulate the following new class of multiobjective fractional variational control problems with interval-valued components, called the Primal Problem (in short, PP):

$$
\begin{gathered}
\min _{(a, u)}\left\{\mathcal{K}(a, u)=\left(\left[\frac{F^{1, L}(a, u)}{G^{1, L}(a, u)}, \frac{F^{1, U}(a, u)}{G^{1, U}(a, u)}\right], \ldots,\left[\frac{F^{p, L}(a, u)}{G^{p, L}(a, u)}, \frac{F^{p}}{G^{p}},\right.\right.\right. \\
\text { subject to } \\
\frac{\partial a^{i}}{\partial t^{\alpha}}(t)=\mathcal{X}_{\alpha}^{i}(t, a(t), u(t)), \quad i=\overline{1, n}, \alpha=\overline{1, m}, t \in \Omega \\
\mathcal{Y}(t, a(t), u(t)) \leq 0, \quad t \in \Omega \\
a\left(t_{1}\right)=a_{1}=\text { given, } \quad a\left(t_{2}\right)=a_{2}=\text { given, }
\end{gathered}
$$

where, for $r=\overline{1, p}$, we have denoted

$$
\begin{gathered}
\frac{F^{r}(a, u)}{G^{r}(a, u)}=\frac{\left[F^{r, L}(a, u), F^{r, U}(a, u)\right]}{\left[G^{r, L}(a, u), G^{r, U}(a, u)\right]}=\left[\frac{F^{r, L}(a, u)}{G^{r, L}(a, u)}, \frac{F^{r, U}(a, u)}{G^{r, U}(a, u)}\right] \\
=\left[\frac{\int_{\Omega} f_{\alpha}^{r, L}(t, a(t), u(t)) d t^{\alpha}}{\int_{\Omega} g_{\alpha}^{r, L}(t, a(t), u(t)) d t^{\alpha}}, \frac{\int_{\Omega} f_{\alpha}^{r, U}(t, a(t), u(t)) d t^{\alpha}}{\int_{\Omega} g_{\alpha}^{r, U}(t, a(t), u(t)) d t^{\alpha}}\right],
\end{gathered}
$$

and it is assumed that $G^{r}(a, u)>[0,0], \forall(a, u) \in \mathcal{A} \times \mathcal{U}$.

The set of all feasible solutions in $(P P)$ is defined by

$$
\mathcal{D}:=\{(a, u) \mid a \in \mathcal{A}, u \in \mathcal{U} \text { satisfying (1), (2), (3) }\} .
$$


Definition 5. A feasible solution $\left(a^{0}, u^{0}\right) \in \mathcal{D}$ in $(P P)$ is called an LU-efficient solution if there is no other $(a, u) \in \mathcal{D}$ such that $\mathcal{K}(a, u) \preceq \mathcal{K}\left(a^{0}, u^{0}\right)$.

Taking into account Treanţă [9], Mititelu and Treanţă [8], and Treanţă and Mititelu [26], under constraint qualification assumptions, if $\left(a^{0}, u^{0}\right) \in \mathcal{D}$ is an LU-efficient solution of the variational control problem $(P P)$, then there exist $\theta=\left(\theta_{r, l}\right), \varphi(t)$, and $\phi(t)$, with $\varphi(t)=$ $\left(\varphi^{\beta}(t)\right), \phi(t)=\left(\phi_{i}^{\alpha}(t)\right)$ piecewise smooth functions, satisfying the following conditions (see Einstein summation)

$$
\begin{gathered}
\theta_{r, \iota}\left[G^{r, l}\left(a^{0}, u^{0}\right) \frac{\partial f_{\alpha}^{r, l}}{\partial a^{i}}\left(t, a^{0}(t), u^{0}(t)\right)-F^{r, l}\left(a^{0}, u^{0}\right) \frac{\partial g_{\alpha}^{r, l}}{\partial a^{i}}\left(t, a^{0}(t), u^{0}(t)\right)\right] \\
+\phi_{i}^{\alpha}(t) \frac{\partial \mathcal{X}_{\alpha}^{i}}{\partial a^{i}}\left(t, a^{0}(t), u^{0}(t)\right)+\varphi^{\beta}(t) \frac{\partial \mathcal{Y}_{\beta}}{\partial a^{i}}\left(t, a^{0}(t), u^{0}(t)\right)+\frac{\partial \phi_{i}^{\alpha}}{\partial t^{\alpha}}(t)=0, \quad i=\overline{1, n}, \iota=L, U \\
\theta_{r, \iota}\left[G^{r, l}\left(a^{0}, u^{0}\right) \frac{\partial f_{\alpha}^{r, l}}{\partial u^{j}}\left(t, a^{0}(t), u^{0}(t)\right)-F^{r, \iota}\left(a^{0}, u^{0}\right) \frac{\partial g_{\alpha}^{r, l}}{\partial u^{j}}\left(t, a^{0}(t), u^{0}(t)\right)\right] \\
+\phi_{i}^{\alpha}(t) \frac{\partial \mathcal{X}_{\alpha}^{i}}{\partial u^{j}}\left(t, a^{0}(t), u^{0}(t)\right)+\varphi^{\beta}(t) \frac{\partial \mathcal{Y}_{\beta}}{\partial u^{j}}\left(t, a^{0}(t), u^{0}(t)\right)=0, \quad j=\overline{1, k}, \iota=L, U \\
\varphi^{\beta}(t) \mathcal{Y}_{\beta}\left(t, a^{0}(t), u^{0}(t)\right)=0 \quad(\text { no summation), } \quad(\theta, \varphi(t)) \succeq 0,
\end{gathered}
$$

for all $t \in \Omega$, except at discontinuities.

Definition 6. The feasible solution $\left(a^{0}, u^{0}\right) \in \mathcal{D}$ is a normal LU-efficient solution for (PP) if the necessary LU-efficiency conditions formulated in (4)-(6) hold for $\theta \succeq 0$ and $e^{t} \theta_{\iota}=1, e^{t}=$ $(1, \ldots, 1) \in \mathbb{R}^{p}, \iota=L, U$.

\section{Mond-Weir Duality}

Let $\left\{\mathcal{Q}_{1}, \mathcal{Q}_{2}, \cdots, \mathcal{Q}_{s}\right\}$ be a partition of the set $\mathcal{Q}=\{1,2, \cdots, q\}$, where $s<q$. For $(b, w) \in \mathcal{A} \times \mathcal{U}$ and $N:=\sum_{\vartheta=1}^{s} \vartheta$, with the same notations as in Section 2, we associate to $(P P)$ the next multiobjective fractional variational control problem with interval-valued components of the vector, called the Dual Problem (in short DP):

$$
\begin{aligned}
& \max _{(b, w)}\left\{\mathcal{K}(b, w)=\left(\left[\frac{\int_{\gamma} f_{\alpha}^{1, L}(t, b(t), w(t)) d t^{\alpha}}{\int_{\gamma} g_{\alpha}^{1, L}(t, b(t), w(t)) d t^{\alpha}}, \frac{\int_{\gamma} f_{\alpha}^{1, U}(t, b(t), w(t)) d t^{\alpha}}{\int_{\gamma} g_{\alpha}^{1, U}(t, b(t), w(t)) d t^{\alpha}}\right],\right.\right. \\
& \left.\left.\ldots,\left[\frac{\int_{\gamma} f_{\alpha}^{p, L}(t, b(t), w(t)) d t^{\alpha}}{\int_{\gamma} g_{\alpha}^{p, L}(t, b(t), w(t)) d t^{\alpha}}, \frac{\int_{\gamma} f_{\alpha}^{p, U}(t, b(t), w(t)) d t^{\alpha}}{\int_{\gamma} g_{\alpha}^{p, U}(t, b(t), w(t)) d t^{\alpha}}\right],\right)\right\}
\end{aligned}
$$

subject to

$$
\begin{gathered}
\theta_{r, \iota}\left[G^{r, l}(b, w) \frac{\partial f_{\alpha}^{r, l}}{\partial b^{i}}(t, b(t), w(t))-F^{r, l}(b, w) \frac{\partial g_{\alpha}^{r, l}}{\partial b^{i}}(t, b(t), w(t))\right] \\
+\phi_{i}^{\alpha}(t) \frac{\partial \mathcal{X}_{\alpha}^{i}}{\partial b^{i}}(t, b(t), w(t))+\varphi^{\beta}(t) \frac{\partial \mathcal{Y}_{\beta}}{\partial b^{i}}(t, b(t), w(t))+\frac{\partial \phi_{i}^{\alpha}}{\partial t^{\alpha}}(t)=0, \quad i=\overline{1, n}, \iota=L, U \\
\theta_{r, l}\left[G^{r, l}(b, w) \frac{\partial f_{\alpha}^{r, l}}{\partial w^{j}}(t, b(t), w(t))-F^{r, l}(b, w) \frac{\partial g_{\alpha}^{r, l}}{\partial w^{j}}(t, b(t), w(t))\right] \\
+\phi_{i}^{\alpha}(t) \frac{\partial \mathcal{X}_{\alpha}^{i}}{\partial w^{j}}(t, b(t), w(t))+\varphi^{\beta}(t) \frac{\partial \mathcal{Y}_{\beta}}{\partial w^{j}}(t, b(t), w(t))=0, \quad j=\overline{1, k}, \iota=L, U \\
\phi_{i}^{\alpha}(t)\left[\mathcal{X}_{\alpha}^{i}(t, b(t), w(t))-\frac{\partial b^{i}}{\partial t^{\alpha}}(t)\right] \frac{\vartheta}{N}+\sum_{\beta \in \mathcal{Q}_{\vartheta}} \varphi^{\beta}(t) \mathcal{Y}_{\beta}(t, b(t), w(t)) \geq 0, \quad \vartheta=\overline{1, s}
\end{gathered}
$$




$$
\theta=\left(\theta_{r, l}\right) \succeq 0, \quad \varphi(t)=\left(\varphi^{\beta}(t)\right) \geq 0, \quad b\left(t_{1}\right)=a_{1}=\text { given, } \quad b\left(t_{2}\right)=a_{2}=\text { given, } \iota=L, U \text {. }
$$

In this section, we establish that the multiobjective optimization problems with interval-valued components of the ratio vector, $(P P)$ and $(D P)$, are a Mond-Weir (see [27]) dual pair under $(\varrho, \psi, \omega)$-quasiinvexity hypotheses. Further, assume that $\triangle$ is the set of all feasible solutions associated with $(D P)$.

Now, in accordance with Treanţă and Mititelu [17], we formulate and prove a first duality result.

Theorem 1 (Weak Duality). Let $(a, u) \in \mathcal{D}$ be a feasible solution of the multiobjective variational control problem with interval-valued components $(P P)$ and $(b, w, \theta, \phi, \varphi) \in \triangle$ be a feasible solution of the multiobjective variational control problem with interval-valued components $(D P)$. Further, assume that the following conditions are fulfilled:

(a) Each functional

$$
\mathcal{F}_{r, l}^{b, w}(a, u)=\int_{\gamma}\left[G^{r, l}(b, w) f_{\alpha}^{r, l}(t, a(t), u(t))-F^{r, \iota}(b, w) g_{\alpha}^{r, l}(t, a(t), u(t))\right] d t^{\alpha}, r=\overline{1, p}, \iota=L, U
$$

is $\left(\varrho^{r, 1}, \psi, \omega\right)$-quasiinvex at $(b, w)$ with respect to $v$ and $\tau$, or, equivalently, each interval-valued path-independent curvilinear integral functional

$$
\mathcal{F}_{r}^{b, w}(a, u)=\left[\mathcal{F}_{r, L}^{b, w}(a, u), \mathcal{F}_{r, U}^{b, w}(a, u)\right], \quad r=\overline{1, p}
$$

is $\left(\varrho^{r, 1}, \psi, \omega\right)$-quasiinvex at $(b, w)$ with respect to $v$ and $\tau$.

(b) For $\vartheta=\overline{1, s}$, each functional

$$
\mathcal{Z}_{\vartheta}(a, u)=\int_{\gamma}\left\{\phi_{i}^{\alpha}(t)\left[\mathcal{X}_{\alpha}^{i}(t, a(t), u(t))-\frac{\partial a^{i}}{\partial t^{\alpha}}(t)\right] \frac{\vartheta}{N}+\sum_{\beta \in \mathcal{Q}_{\vartheta}} \varphi^{\beta}(t) \mathcal{Y}_{\beta}(t, a(t), u(t))\right\} d t^{\alpha}
$$

is $\left(\varrho^{\vartheta, 2}, \psi, \omega\right)$-quasiinvex at $(b, w)$ with respect to $v$ and $\tau$.

(c) At least one of the functionals given in $(a),(b)$ is strictly $(\varrho, \psi, \omega)$-quasiinvex at $(b, w)$ with respect to $v$ and $\tau$, where $\varrho=\varrho^{r, 1}$ or $\varrho^{\vartheta, 2}$.

(d) $\theta_{r, \iota} \varrho^{r, 1}+\sum_{\vartheta=1}^{s} \varrho^{\vartheta, 2} \geq 0 \quad\left(\varrho^{r, 1}, \varrho^{\vartheta, 2} \in \mathbb{R}\right), \iota=L, U$.

Then, the infimum of $(P P)$ is greater than or equal to the supremum of $(D P)$.

Proof. Denote by $\pi(a, u)$ and $\delta(b, w, \theta, \phi, \varphi)$ the value of problem $(P P)$ at $(a, u) \in \mathcal{D}$ and the value of problem $(D P)$ at $(b, w, \theta, \phi, \varphi) \in \triangle$, respectively. Contrary to the result, suppose that $\pi(a, u) \preceq \delta(b, w, \theta, \phi, \varphi)$. Further, for $r=\overline{1, p}, \iota=L, U$ and $\vartheta=\overline{1, s}$, consider the following nonempty set:

$$
S=\left\{(a, u) \in \mathcal{A} \times \mathcal{U} \mid \mathcal{F}_{r, l}^{b, w}(a, u) \leq \mathcal{F}_{r, l}^{b, w}(b, w), \mathcal{Z}_{\vartheta}(a, u) \leq \mathcal{Z}_{\vartheta}(b, w)\right\} .
$$

Using $(a)$ for $(a, u) \in S$ and $r=\overline{1, p}, \iota=L, U$, we get

$$
\begin{gathered}
\mathcal{F}_{r, l}^{b, w}(a, u) \leq \mathcal{F}_{r, l}^{b, w}(b, w) \Longrightarrow \\
\psi(a, u, b, w) \int_{\gamma}\left[G^{r, l}(b, w)\left(f_{\alpha}^{r, l}\right)_{b}(t, b(t), w(t))-F^{r, l}(b, w)\left(g_{\alpha}^{r, l}\right)_{b}(t, b(t), w(t))\right] v d t^{\alpha} \\
+\psi(a, u, b, w) \int_{\gamma}\left[G^{r, l}(b, w)\left(f_{\alpha}^{r, l}\right)_{w}(t, b(t), w(t))-F^{r, l}(b, w)\left(g_{\alpha}^{r, l}\right)_{w}(t, b(t), w(t))\right] \tau d t^{\alpha} \\
\leq-\varrho^{r, 1} \psi(a, u, b, w) \omega^{2}((a, u),(b, w)) .
\end{gathered}
$$


Multiplying by $\theta=\left(\theta_{r, \iota}\right) \succeq 0, \iota=L, U$, and making summation over $r=\overline{1, p}$, we find

$$
\begin{gathered}
\psi(a, u, b, w) \int_{\gamma} \theta_{r, l}\left[G^{r, l}(b, w)\left(f_{\alpha}^{r, l}\right)_{b}(t, b(t), w(t))-F^{r, l}(b, w)\left(g_{\alpha}^{r, l}\right)_{b}(t, b(t), w(t))\right] v d t^{\alpha} \\
+\psi(a, u, b, w) \int_{\gamma} \theta_{r, l}\left[G^{r, l}(b, w)\left(f_{\alpha}^{r, l}\right)_{w}(t, b(t), w(t))-F^{r, l}(b, w)\left(g_{\alpha}^{r, l}\right)_{w}(t, b(t), w(t))\right] \tau d t^{\alpha} \\
\leq-\theta_{r, l} \varrho^{r, 1} \psi(a, u, b, w) \omega^{2}((a, u),(b, w)) .
\end{gathered}
$$

For $(a, u) \in S$, the inequality $\mathcal{Z}_{\vartheta}(a, u) \leq \mathcal{Z}_{\vartheta}(b, w)$ holds and, according to $(b)$ and making summation over $\vartheta=\overline{1, s}$, it follows that

$$
\begin{gathered}
\psi(a, u, b, w) \int_{\gamma}\left[\phi_{i}^{\alpha}(t)\left(\mathcal{X}_{\alpha}^{i}\right)_{b}(t, b(t), w(t)) v-\phi^{\alpha}(t) D_{\alpha} v+\phi_{i}^{\alpha}(t)\left(\mathcal{X}_{\alpha}^{i}\right)_{w}(t, b(t), w(t)) \tau\right] d t^{\alpha} \\
+\psi(a, u, b, w) \int_{\gamma}\left[\varphi^{\beta}(t)\left(\mathcal{Y}_{\beta}\right)_{b}(t, b(t), w(t)) v+\varphi^{\beta}(t)\left(\mathcal{Y}_{\beta}\right)_{w}(t, b(t), w(t)) \tau\right] d t^{\alpha} \\
\leq-\sum_{\vartheta=1}^{s} \varrho^{\vartheta, 2} \psi(a, u, b, w) \omega^{2}((a, u),(b, w)) .
\end{gathered}
$$

Making the sum $(11)+(12)$ side by side and taking into account $(c)$, we have

$$
\begin{gathered}
\psi(a, u, b, w) \int_{\gamma} \theta_{r, l}\left[G^{r, l}(b, w)\left(f_{\alpha}^{r, l}\right)_{b}(t, b(t), w(t))-F^{r, l}(b, w)\left(g_{\alpha}^{r, l}\right)_{b}(t, b(t), w(t))\right] v d t^{\alpha} \\
+\psi(a, u, b, w) \int_{\gamma}\left[\phi_{i}^{\alpha}(t)\left(\mathcal{X}_{\alpha}^{i}\right)_{b}(t, b(t), w(t))+\varphi^{\beta}(t)\left(\mathcal{Y}_{\beta}\right)_{b}(t, b(t), w(t))\right] v d t^{\alpha} \\
+\psi(a, u, b, w) \int_{\gamma} \theta_{r, l}\left[G^{r, l}(b, w)\left(f_{\alpha}^{r, l}\right)_{w}(t, b(t), w(t))-F^{r, l}(b, w)\left(g_{\alpha}^{r, l}\right)_{w}(t, b(t), w(t))\right] \tau d t^{\alpha} \\
+\psi(a, u, b, w) \int_{\gamma}\left[\phi_{i}^{\alpha}(t)\left(\mathcal{X}_{\alpha}^{i}\right)_{w}(t, b(t), w(t))+\varphi^{\beta}(t)\left(\mathcal{Y}_{\beta}\right)_{w}(t, b(t), w(t))\right] \tau d t^{\alpha} \\
-\psi(a, u, b, w) \int_{\gamma}\left[\phi^{\alpha}(t) D_{\alpha} v\right] d t^{\alpha}<-\left(\theta_{r, \iota} \varrho^{r, 1}+\sum_{\vartheta=1}^{s} \varrho^{\vartheta, 2}\right) \psi(a, u, b, w) \omega^{2}((a, u),(b, w)), \iota=L, U .
\end{gathered}
$$

The previous inequality implies $\psi(a, u, b, w)>0$ and, as a consequence, we can rewrite it as

$$
\begin{gathered}
\int_{\gamma} \theta_{r, \iota}\left[G^{r, l}(b, w)\left(f_{\alpha}^{r, l}\right)_{b}(t, b(t), w(t))-F^{r, l}(b, w)\left(g_{\alpha}^{r, l}\right)_{b}(t, b(t), w(t))\right] v d t^{\alpha} \\
+\int_{\gamma}\left[\phi_{i}^{\alpha}(t)\left(\mathcal{X}_{\alpha}^{i}\right)_{b}(t, b(t), w(t))+\varphi^{\beta}(t)\left(\mathcal{Y}_{\beta}\right)_{b}(t, b(t), w(t))\right] v d t^{\alpha} \\
+\int_{\gamma} \theta_{r, \iota}\left[G^{r, l}(b, w)\left(f_{\alpha}^{r, l}\right)_{w}(t, b(t), w(t))-F^{r, l}(b, w)\left(g_{\alpha}^{r, l}\right)_{w}(t, b(t), w(t))\right] \tau d t^{\alpha} \\
+\int_{\gamma}\left[\phi_{i}^{\alpha}(t)\left(\mathcal{X}_{\alpha}^{i}\right)_{w}(t, b(t), w(t))+\varphi^{\beta}(t)\left(\mathcal{Y}_{\beta}\right)_{w}(t, b(t), w(t))\right] \tau d t^{\alpha} \\
-\int_{\gamma}\left[\phi^{\alpha}(t) D_{\alpha} v\right] d t^{\alpha}<-\left(\theta_{r, \iota} \varrho^{r, 1}+\sum_{\vartheta=1}^{s} \varrho^{\vartheta, 2}\right) \omega^{2}((a, u),(b, w)), \quad \iota=L, U .
\end{gathered}
$$

Now, considering constraints $(7)$ and $(8)$ of $(D P)$, we obtain $\left.-\int_{\gamma} v D_{\alpha} \phi^{\alpha}(t) d t^{\alpha}-\int_{\gamma}\left[\phi^{\alpha}(t) D_{\alpha} v\right] d t^{\alpha}<-\left(\theta_{r, \iota} \varrho^{r, 1}+\sum_{\vartheta=1}^{s} \varrho^{\vartheta, 2}\right)\right) \omega^{2}((a, u),(b, w)), \iota=L, U$.

By direct computation, we get

$$
D_{\alpha}\left[v \phi^{\alpha}(t)\right]=\phi^{\alpha}(t) D_{\alpha} v+v D_{\alpha} \phi^{\alpha}(t)
$$




$$
\int_{\gamma} v D_{\alpha} \phi^{\alpha}(t) d t^{\alpha}=\int_{\gamma} D_{\alpha}\left[v \phi^{\alpha}(t)\right] d t^{\alpha}-\int_{\gamma}\left[\phi^{\alpha}(t) D_{\alpha} v\right] d t^{\alpha}
$$

but, applying the condition $v\left(t_{1}\right)=v\left(t_{2}\right)=0$ and the result " $A$ total divergence is equal to $a$ total derivative.", we get

$$
\int_{\Gamma} D_{\alpha}\left[v \phi^{\alpha}(t)\right] d t^{\alpha}=0 .
$$

It results that

$$
-\int_{\gamma} v D_{\alpha} \phi^{\alpha}(t) d t^{\alpha}-\int_{\gamma}\left[\phi^{\alpha}(t) D_{\alpha} v\right] d t^{\alpha}=0 .
$$

Consequently,

$$
0<-\left(\theta_{r, \iota} \varrho^{r, 1}+\sum_{\vartheta=1}^{s} \varrho^{\vartheta, 2}\right) \omega^{2}((a, u),(b, w)), \quad \iota=L, U
$$

and applying the hypothesis $(d)$ and $\omega^{2}((a, u),(b, w)) \geq 0$, we get a contradiction. Therefore, the infimum of $(P P)$ is greater than or equal to the supremum of $(D P)$.

The next, according to Treanţă and Mititelu [17], establishes a strong duality between the two considered multiobjective optimization problems with interval-valued components.

Theorem 2 (Strong Duality). Under the same $(\varrho, \psi, \omega)$-quasiinvexity hypotheses formulated in Theorem 1, if $\left(a^{0}, u^{0}\right) \in \mathcal{D}$ is a normal LU-efficient solution of the Primal Problem (PP), then there exist $\theta^{0}, \varphi^{0}(t)$, and $\phi^{0}(t)$ such that $\left(a^{0}, u^{0}, \theta^{0}, \phi^{0}, \varphi^{0}\right) \in \triangle$ is an LU-efficient solution of the Dual Problem (DP) and the corresponding objective values are equal.

Proof. Considering that $\left(a^{0}, u^{0}\right) \in \mathcal{D}$ is a normal LU-efficient solution in $(P P)$, the necessary LU-efficiency conditions, formulated in (4)-(6), involve that there exist $\theta^{0}, \varphi^{0}(t)$, and $\phi^{0}(t)$ such that $\left(a^{0}, u^{0}, \theta^{0}, \phi^{0}, \varphi^{0}\right)$ is a feasible solution for $(D P)$. Since

$$
\frac{\partial a^{0 i}}{\partial t^{\alpha}}(t)=\mathcal{X}_{\alpha}^{i}\left(t, a^{0}(t), u^{0}(t)\right), \quad i=\overline{1, n}, \alpha=\overline{1, m}, t \in \Omega
$$

and (by (6))

$$
\varphi^{\beta}(t) \mathcal{Y}_{\beta}\left(t, a^{0}(t), u^{0}(t)\right)=0, \quad(\text { summation over } \beta), \quad t \in \Omega,
$$

the dual objective has the same value as the primal objective and, by Theorem $1,\left(a^{0}, u^{0}, \theta^{0}\right.$, $\left.\phi^{0}, \varphi^{0}\right) \in \triangle$ is an LU-efficient solution of $(D P)$.

The following theorem formulates a converse duality result associated with the considered multiobjective optimization problems with interval-valued components.

Theorem 3 (Converse Duality). Let $\left(a^{0}, u^{0}, \theta^{0}, \phi^{0}, \varphi^{0}\right) \in \triangle$ be an LU-efficient solution of $(D P)$. Further, assume that the following conditions are fulfilled:

(a) $(\bar{a}, \bar{u}) \in \mathcal{D}$ is a normal LU-efficient solution of $(P P)$;

(b) the hypotheses of Theorem 1 are satisfied for $\left(a^{0}, u^{0}, \theta^{0}, \phi^{0}, \varphi^{0}\right)$.

Then, $(\bar{a}, \bar{u})=\left(a^{0}, u^{0}\right)$ and the corresponding objective values are equal.

Proof. Contrary to the result, let us suppose that $\left(a^{0}, u^{0}\right)$ is not a normal LU-efficient solution of $(P P)$, that is, $(\bar{a}, \bar{u}) \neq\left(a^{0}, u^{0}\right)$. As $(\bar{a}, \bar{u}) \in \mathcal{D}$ is a normal LU-efficient solution of $(P P)$, according to Treanţă [9] and Mititelu and Treanţă [8], there exist $\bar{\theta}, \bar{\varphi}(t)$ and $\bar{\phi}(t)$, satisfying (4)-(6) and Definition 6. It follows

$$
\bar{\phi}_{i}^{\alpha}(t)\left[\mathcal{X}_{\alpha}^{i}(t, \bar{a}(t), \bar{u}(t))-\frac{\partial \bar{a}^{i}}{\partial t^{\alpha}}(t)\right] \frac{\vartheta}{N}+\sum_{\beta \in \mathcal{Q}_{\theta}} \bar{\varphi}^{\beta}(t) \mathcal{Y}_{\beta}(t, \bar{a}(t), \bar{u}(t)) \geq 0, \quad \vartheta=\overline{1, s}
$$


and, therefore, $(\bar{a}, \bar{u}, \bar{\theta}, \bar{\phi}, \bar{\varphi}) \in \triangle$. Moreover, we have $\pi(\bar{a}, \bar{u})=\delta(\bar{a}, \bar{u}, \bar{\theta}, \bar{\phi}, \bar{\varphi})$. In accordance to Theorem 1 , we have $\pi(\bar{a}, \bar{u}) \npreceq \delta\left(a^{0}, u^{0}, \theta^{0}, \phi^{0}, \varphi^{0}\right)$ or $\delta(\bar{a}, \bar{u}, \bar{\theta}, \bar{\phi}, \bar{\varphi}) \npreceq \delta\left(a^{0}, u^{0}, \theta^{0}\right.$, $\left.\phi^{0}, \varphi^{0}\right)$. This contradicts the maximal LU-efficiency of $\left(a^{0}, u^{0}, \theta^{0}, \phi^{0}, \varphi^{0}\right)$. Hence, $(\bar{a}, \bar{u})=$ $\left(a^{0}, u^{0}\right)$ and the corresponding objective values are equal.

Remark 1. If, for $r=\overline{1, p}$ and $(a, u) \in \mathcal{A} \times \mathcal{U}$, each interval-valued path-independent curvilinear integral functional $\int_{\gamma} g_{\alpha}^{r}(t, a(t), u(t)) d t^{\alpha}$ is equal to 1 , then we obtain primal and dual multiobjective nonfractional variational control problems with interval-valued components and the corresponding Mond-Weir duality results.

\section{Conclusions}

In this paper, we have studied a dual pair of multiobjective variational control problems with interval-valued components. More precisely, based on the new notion of $(\varrho, \psi, \omega)$ quasiinvexity associated with interval-valued path-independent curvilinear integral functionals, we have established weak, strong, and converse duality results for the considered class of optimization problems. Moreover, by considering the physical meaning of the curvilinear integrals (mechanical work) and the importance of Interval Analysis in the applied sciences and engineering, this research work can be seen as a starting point for further investigations.

Funding: This research received no external funding.

Institutional Review Board Statement: Not applicable.

Informed Consent Statement: Not applicable.

Data Availability Statement: Not applicable.

Acknowledgments: The author would like to thank anonymous referees for their careful reading and constructive suggestions that improve substantially the revision of the manuscript.

Conflicts of Interest: The author declares no conflict of interest.

\section{References}

1. Hanson, M.A. On sufficiency of Kuhn-Tucker conditions. J. Math. Anal. Appl. 1981, 80, 545-550. [CrossRef]

2. Mond, B.; Hanson, M.A. Duality for control problems. SIAM J. Control 1968, 6, 114-120. [CrossRef]

3. Mond, B.; Smart, I. Duality and sufficiency in control problems with invexity. J. Math. Anal. Appl. 1988, 136, 325-333. [CrossRef]

4. Aggarwal, S.; Bhatia, D.; Neelam, L. Duality in multiple right hand choice linear fractional problems. J. Inform. Optim. Sci. 1991, 12, 13-24. [CrossRef]

5. Mishra, S.K.; Mukherjee, R.N. Multiobjective control problem with V-invexity. J. Math. Anal. Appl. 1999, 235, 1-12. [CrossRef]

6. Ahmad, I.; Sharma, S. Sufficiency and duality for multiobjective variational control problems with generalized $(F, \alpha, \rho, \theta)$-Vconvexity. Nonlinear Anal. 2010, 72, 2564-2579. [CrossRef]

7. Antczak, T. Duality for multiobjective variational control problems with $(\Phi, \rho)$-invexity. Calcolo 2014, 51, 393-421. [CrossRef]

8. Mititelu, S.; Treanţă, S. Efficiency conditions in vector control problems governed by multiple integrals. J. Appl. Math. Comput. 2018, 57, 647-665. [CrossRef]

9. Treanţă, S. Efficiency in uncertain variational control problems. Neural Comput. Appl. 2020. [CrossRef]

10. Liang, Z; Ye, Q. Duality for a class of multiobjective control problems with generalized invexity. J. Math. Anal. Appl. 2001, 256, 446-461.

11. Mititelu, S. Efficiency conditions for multiobjective fractional variational problems. Appl. Sci. 2008, 10, 162-175.

12. Treanţă, S.; Udrişte, C. On efficiency conditions for multiobjective variational problems involving higher order derivatives. In Proceedings of the 15th International Conference on Automatic Control, Modelling \& Simulation (ACMOS'13), Brasov, Romania, 1-3 June 2013; pp. 157-164.

13. Zalmai, G.J. Generalized $(\mathcal{F}, b, \phi, \rho, \theta)$-univex $n$-set functions and semiparametric duality models in multiobjective fractional subset programming. Int. J. Math. Math. Sci. 2005, 6, 949-973. [CrossRef]

14. Hachimi, M.; Aghezzaf, B. Sufficiency and duality in multiobjective variational problems with generalized type I functions. J. Glob. Optim. 2006, 34, 191-218. [CrossRef]

15. Treanţă, S. On a vector optimization problem involving higher order derivatives. UPB. Sci. Bull. Ser. A 2015, 77, 115-128.

16. Treanţă, S. Multiobjective fractional variational problem on higher-order jet bundles. Commun. Math. Stat. 2016, 4, 323-340. [CrossRef] 
17. Treanţă, S.; Mititelu, S. Duality with $(\rho, b)$-quasiinvexity for multidimensional vector fractional control problems. J. Inform. Optim. Sci. 2019, 40, 1429-1445. [CrossRef]

18. Chen, X. Duality for a class of multiobjective control problems. J. Math. Anal. Appl. 2002, 267, 377-394.

19. Kim, D.S.; Kim, A.L. Optimality and duality for nondifferentiable multiobjective variational problems. J. Math. Anal. Appl. 2002, 274, 255-278. [CrossRef]

20. Gulati, T.R.; Husain, I.; Ahmed, A. Optimality conditions and duality for multiobjective control problems. J. Appl. Anal. 2005, 11, 225-245. [CrossRef]

21. Nahak, C.; Nanda, S. Sufficient optimality criteria and duality for multiobjective variational control problems with $V$-invexity. Nonlinear Anal. 2007, 66, 1513-1525. [CrossRef]

22. Arana-Jiménez, M.; Ruiz-Garzón, G.; Rufián-Lizana, A.; Osuna-Gómez, R. A necessary and sufficient condition for duality in multiobjective variational problems. Eur. J. Oper. Res. 2010, 201, 672-681. [CrossRef]

23. Khazafi, K.; Rueda, N.; Enflo, P. Sufficiency and duality for multiobjective control problems under generalized $(B, \rho)$-type I functions. J. Glob. Optim. 2010, 46, 111-132. [CrossRef]

24. Zhang, J.; Liu, S.; Li, L.; Feng, Q. Sufficiency and duality for multiobjective variational control problems with G-invexity. Comput. Math. Appl. 2012, 63, 838-850. [CrossRef]

25. Treanţă, S.; Arana-Jiménez, M. KT-pseudoinvex multidimensional control problem. Optim. Control Appl. Meth. 2018, 39, 1291-1300. [CrossRef]

26. Treanţă, S.; Mititelu, S. Efficiency for variational control problems on Riemann manifolds with geodesic quasiinvex curvilinear integral functionals. Rev. Real Acad. Cienc. Exactas Físicas Naturales Ser. A Matemáticas 2020, 114, 113. [CrossRef]

27. Mond, B.; Weir, T. Generalized concavity and duality. In Generalized Concavity in Optimization and Economics; Schaible, S., Ziemba, W.T., Eds.; Academic Press: New York, NY, USA, 1981; pp. 263-279. 